\title{
ACTITUD HACIA LAS INICIATIVAS DE AYUDA SOCIAL Y SU EFECTO EN LA INTENCIÓN DE REALIZAR DONATIVOS POR PARTE DE LOS HABITANTES DE LA CIUDAD DE GUAYAQUIL
}

\section{ATTITUDE TOWARDS SOCIAL AID INITIATIVES AND THEIR EFFECT ON THE INTENTION TO MAKE DONATIONS BY THE INHABITANTS OF THE CITY OF GUAYAQUIL}

\author{
Gisell Cristina Cedillo Quito ${ }^{1}$, Angie Melany Márquez Veliz² ${ }^{2}$ Ronald \\ Campoverde Aguirre ${ }^{3}$
}

\begin{abstract}
Resumen
El presente estudio busca conocer si la actitud hacia la ayuda a otros individuos o la actitud hacia la ayuda a organizaciones de ayuda social poseen un efecto mayor en la intención de realizar donativos por parte de los habitantes de Guayaquil, teniendo en cuenta el aumento de organizaciones no lucrativas. Se aplicaron cuestionarios para ampliar la perspectiva tomando en consideración dos constructos: Actitud para ayudar a los demás y Actitud hacia las organizaciones benéficas de Webb, Green y Brashear (2000). Además, de variables como la motivación y el destino de los donativos. Como resultados de la búsqueda y metodología aplicada se obtuvo que los habitantes de la ciudad de Guayaquil poseen mayor aceptación a ayudar por medio de organizaciones benéficas a las diferentes causas, su mayor motivación a dar donativos se da cuando existe una emergencia, sí su destino es a fundaciones que ayudan a personas con enfermedades terminales. También, reflejó que el género femenino tiene mayor intención a donar. Se concluye que las organizaciones benéficas a pesar de tener una gran aceptación por parte del público no deben descuidar la imagen positiva que ya poseen.
\end{abstract}

Códigos JEL: I31, L31

\footnotetext{
${ }^{1}$ Escuela Superior Politécnica del Litoral, Facultad de Ciencias Sociales y Humanísticas, (Ecuador).

E-mail: gccedill@espol.edu.ec

${ }^{2}$ Escuela Superior Politécnica del Litoral, Facultad de Ciencias Sociales y Humanísticas, (Ecuador).

E-mail: angmemar@espol.edu.ec

${ }^{3}$ Escuela Superior Politécnica del Litoral, Facultad de Ciencias Sociales y Humanísticas, (Ecuador).

E-mail: recampov@espol.edu.ec
} 


\begin{abstract}
Keywords:
donation, intent, social initiative, NGO
\end{abstract}

\section{INTRODUCCIÓN}

El Ecuador siempre ha estado influenciado por las luchas sociales en busca de una ciudadanía activa, lo que se refleja en distintas organizaciones sociales y ciudadanas. Según el directorio del Sistema Unificado de Información de Organizaciones Sociales (SUIOS) existen 65 923 organizaciones totales en el país, alrededor de 2229 fundaciones registradas y un aproximado de 9582 organizaciones en la provincia del Guayas durante el año 2019.

Como ya se mencionó existe un considerable número de fundaciones, asociaciones $\mathrm{u}$ organizaciones sin fines de lucro. Según el Directorio ONG 2019, Ministerio de Relaciones y Movilidad Humana alrededor de 271 organizaciones extranjeras están registradas en la Secretaría Técnica de Cooperación Nacional. Es decir, estas organizaciones extranjeras han estado interesadas e incluso han realizado actividades de cooperación internacional no reembolsable en el Ecuador. Por lo tanto, es notable que las ONG desempeñan un papel cada vez más importante, pues buscan la construcción de una sociedad solidaria y equitativa en la que todos sus ciudadanos lleven una vida digna.
El nacimiento de las fundaciones sin fines de lucro surge como una iniciativa de ayuda social llevada a cabo por la ciudadanía, que se propone ayudar a superar las dificultades que el individuo tiene para cubrir sus necesidades básicas, así como para colaborar con el Estado para garantizar una vida digna. Alguna de las actividades que realizan estas organizaciones son el diseño y la implantación de estrategias para la defensa por causas justas, sociales, ambientales, laborales, etc.; el desarrollo de proyectos de carácter económico y/o culturales para el desarrollo de las diferentes comunidades, así como en ocasiones aportes económicos directos (AldamizEcheverría, 2003).

Estas organizaciones se financian con donaciones hechas por el público, las empresas, el gobierno o por la venta de productos manuales o artesanales. Cuyo objetivo es brindar una ayuda a las personas de escasos recursos de un sector marginal. Su principal característica se basa en un liderazgo comunitario y autónomo, en el cual, su fuerza laboral se conforma por personas que se ofrecen voluntariamente a ayudar sin recibir una recompensación monetaria o algún tipo de reconocimiento. La mayoría de estas organizaciones pertenecen al sector privado, como la Junta de Beneficencia de Guayaquil, 
como la Sociedad de Beneficencia de Señoras, entre otras.

Como se mencionó anteriormente, las organizaciones sin fines de lucro inicialmente se financian a través de donaciones. La donación es el acto que realiza una persona, denominada donante, para ayudar a los necesitados a tener una vida más equitativa, de forma voluntaria, sin recibir ni esperar distinción alguna. Es decir, los donantes traspasan sus bienes, dinero u órganos a un individuo que está pasando una carencia, necesidad o que su vida dependa de dicha la donación. Hay diversos tipos de donantes y varias maneras de realizar donaciones que dependen del tipo de organización. Por ejemplo, los donantes de sangre contribuyen con la Cruz Roja: los donantes de fondos monetarios, víveres, medicamentos, etc., apoyan a las organizaciones de caridad, maltrato intrafamiliar, educación, asilos, orfanatos, entre otras instituciones no lucrativas.

Un donante nace de la necesidad de contribuir con la sociedad, por medio de la ayuda social que se brinda a las personas que poseen menos recursos o tienen algún tipo de desventaja en comparación con otros grupos de personas. La ayuda social es una acción realizada por el ser humano para mejorar la calidad de vida de su prójimo, es una lucha constante ante las desigualdades sociales, pobreza, violencia o contra cualquier agente externo que afecte a los derechos humanos.

En la actualidad las organizaciones no lucrativas son parte fundamental para la sociedad, ya que se constituye por medio de una iniciativa de ayuda y contribución a la búsqueda de la igualdad. Por este motivo se ven muchas fundaciones, asociaciones y organizaciones en Guayaquil.

$\mathrm{Al}$ existir muchas fundaciones no lucrativas, el financiamiento de ellas se ve afectado ya que tienen que enfocarse en la captación de diferentes donaciones y a su vez estar conscientes de que existen otras instituciones no lucrativas que también buscan captar recursos monetarios; esta preocupación por parte de las fundaciones se puede cubrir por medio de un estudio que analice la actitud hacia las iniciativas de ayuda social y la intención de dar donaciones por parte de los habitantes de Guayaquil, lo que logrará conocer que factores tienen un efecto mayor en la intención de dar donativos monetarios por parte de los ciudadanos de Guayaquil. De esta manera, las fundaciones podrán enfocar sus esfuerzos a esos factores y así captar la mayor cantidad de donativos monetarios.

El motivo de realizar la presente investigación es conocer la actitud que tiene los seres humanos hacia las iniciativas de ayuda social y saber si tiene o no, la intención de realizar donativos monetarios a personas vulnerables o alguna fundación benéfica que se encarga de ayudar a este sector de Guayaquil. Es decir, a personas que se encuentran vulnerables, expuestas y $\sin$ la capacidad de satisfacer sus necesidades básicas. Se pretende ayudar a las fundaciones en especial para que conozcan los motivos por y la razón por la cual el ciudadano guayaquileño va a generar este tipo de ayuda y de tal forma, generar de manera indirecta una ayuda a dicho sector vulnerable, con el fin de mejorar su calidad de vida.

\section{MARCO TEÓRICO}

\section{Actitud hacia las iniciativas de ayuda social}

El concepto de ayuda social sobre el cual vamos a orientar nuestra investigación recoge la información desarrollada por Ander-Egg, Ezequiel (1986) definió a Ayuda social como los trabajos de beneficencia o de asistencia aplicados a personas o grupo de personas que no están en condiciones de satisfacer sus necesidades básicas, tales como alimentación, vestimenta, salud y educación, que son esenciales para alcanzar una calidad de vida adecuada. La ayuda social, principalmente, está dirigida a niños, adolescentes y mujeres vulnerables con el fin de otorgarles herramientas adecuadas que les permita mantener una vida digna y de gozar de sus ejercicios de sus derechos.

La ayuda social nace de personas con actitud a asistir a personas que para ellos son vulnerables o que tienes menor recurso, la actitud es una tendencia que el ser humano aprende para poder responder de una manera positiva o negativa ante 
una actividad u objeto (Fishbein y Ajzen, 1975; Oskamp, 1977). De esta manera cada ser humano tenemos diferentes tipos de actitudes, es decir actitud hacia la donación, al aborto, a ayudar a los demás, a la práctica de un deporte, etc. Estás actitudes están relacionada de manera directa con el comportamiento del ser humano y del entorno que los rodea.

La actitud hacia la donación nace de la necesidad de contribuir con la sociedad, por medio de la ayuda social que se brinda a las personas que poseen menos recursos o tienen algún tipo de desventaja en comparación con otros grupos de personas. La ayuda social es una acción realizada por el ser humano para mejorar la calidad de vida de su prójimo, es una lucha constante ante las desigualdades sociales, pobreza, violencia o contra cualquier agente externo que afecte a los derechos humanos. La mejor manera de ayudar es no afectar la calidad de vida de las personas que nos rodea, generando donaciones voluntarias a las organizaciones que realizan actividades con enfoque social.

Los donadores siempre buscan una organización que los representa, es decir tienen el mismo objetivo, creencia religiosa en las que predominan los mismos valores, tienen las mismas motivaciones. Salconer (1999) y Tachizawa (2007) mencionó que las fuentes más comunes de recaudar recursos financieros son las empresas de cooperación, venta de productos o servicios, organismos del estado, empresas privadas, fundaciones y principalmente por los donantes individuales.

\section{Intención de realizar donativos monetarios}

Al consolidarse en actores sociales, políticos y económicos, las ONG han despertado el interés de los ciudadanos incentivándolos a contribuir con ellas. Debido a esto, se analiza la intención de realizar donativos monetarios. Termino que se caracteriza como el acto mental de acciones en el futuro que poseen un carácter indicativo.

El interés en esta sección es exponer los fenómenos sociales a través, del modelo de dinámica social con influencia cultural. Este modelo explica como los fenómenos sociales pueden ser modelados de manera matemática y por medio de la física. El modelo propuesto por Axelrod (1997a, 1997b) empieza con el planteamiento de la siguiente interrogante: "Si cuando las personas interaccionan y se relacionan tienden a hacerse más parecidas en sus creencias, actitudes y comportamientos. ¿Por qué no desaparecen con el tiempo las diferencias culturales?". Para responder la pregunta se propuso un modelo simple que examina la tendencia hacia una cultura homogénea o globalizada y la persistencia de la diversidad cultural en los sistemas sociales. El modelo de Axelrod delimita la cultura como la mezcla de rasgos culturales, tales como el lenguaje, religión, gustos musicales, postura política, motivaciones, actitudes, comportamientos, etc. Es imprescindible que estos rasgos culturales se modifiquen por medio de las interacciones sociales.

El modelo se basa en dos premisas:

1. Probabilidad de interacción entre individuos es proporcional al número de atributos culturales compartidos.

2. Interacción aumenta la similitud cultural entre individuos. Además, considera un sistema social compuesto por $\mathrm{N}$ individuos distribuidos en el espacio.

El modelo de Axelrod permite un análisis condensado en las propiedades colectivas del sistema social, como resultado de la interacción de los individuos y la influencia social, por lo tanto, este modelo es de gran utilidad para el estudio de análisis sociales, donde los individuos expuestos a diferentes culturas adaptan ciertas costumbres.

\section{Actitud sobre la ayuda social y su intención de donación}

Como se mencionó anteriormente es vital para el análisis cuantitativo de la investigación conocer la actitud de los ciudadanos de Guayaquil, respecto a la ayuda social, cuyo fin es medir su intención para contribuir de forma económica con las organizaciones. Por consiguiente, en el presente trabajo de 
investigación se analizará las características personales, valores, motivaciones y actitudes de las personas que realizan donativos. Además, se estudiará la intención de los individuos para contribuir con donaciones a las entidades no lucrativas, a través del estudio y medición de posibles factores extrínsecos e intrínsecos que influyan en los mismos. Entre las variables propuestas en la investigación se tiene: Gratitud de vida, edad, sexo, nivel de estudio y estatus social.

Entre los modelos de estudios más comunes aplicados en el análisis de la intención de los donantes tenemos los siguientes:

1. El modelo presentado por Sargeant (2009), está construido por entradas como personas beneficiadas, predisposición a donar, maneras de pedir y entregar ayuda e información que llama la atención del donante. Luego indican los factores extrínsecos e intrínsecos que influyen en el donante, en el extrínseco se encuentra la edad, el género y los ingresos, en el intrínseco se encuentra la necesidad de autosatisfacción, de autorrealización, social y de prestigio. Por último, se analiza la percepción del donante sobre los factores antes mencionados y se concluye en la realización o no de la donación.

2. El modelo de estudio de la motivación del voluntariado de tres dimensiones: motivación altruista, egoísta y motivo social es propuesto por Snyder y Cantor (2008).

3. El modelo que estudia el modo y la fuerza de la actitud que influye en el comportamiento de ayuda, propuesto por Briggs (2007).

4. El modelo de actitudes que influyen en las donaciones monetarias a organizaciones benéficas propuesto por Webb, Green, y Brashear (2000), está compuesto por dos secciones como: actitud para ayudar a los demás y actitud hacia las organizaciones benéficas y en cada sección tiene entre 4 y 5 preguntas respectivamente, propone realizar las puntuaciones en una escala Likert del 1 al 7. Siendo este modelo el más indicado para aplicar en el análisis del tema propuesto, porque tiene la cantidad de preguntas adecuadas y que se ajusta a la necesidad de conseguir información relevante para la investigación.

\section{Marco teórico propuesto}

H1: La actitud hacia la ayuda a los demás tiene un efecto mayor en la intención de realizar donativos monetarios.

$\mathrm{H} 2$ : La actitud hacia las organizaciones no lucrativas tiene un efecto mayor en la intención de realizar donativos monetarios.

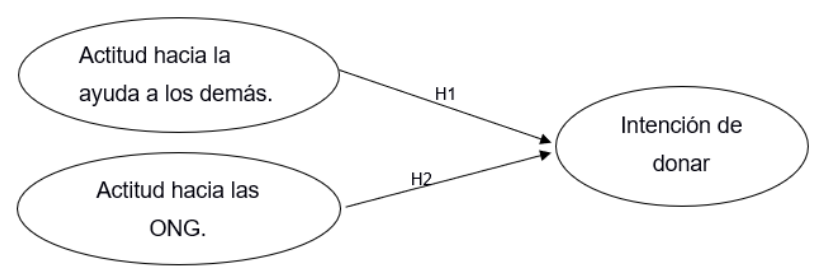

Figura 1. Marco teórico propuesto

\section{METODOLOGÍA}

Como se indicó al inicio en el presente trabajo tiene como principal objetivo analizar la intención de donación de los ciudadanos guayaquileños, para conseguir los datos deseados se procedió a buscar modelos e información que ayude con la investigación planteada. Siendo la población objetivo las personas dispuestas a realizar donativos y que posea alguna fuente de ingreso.

Modelo e Hipótesis de la investigación:

H1: La actitud hacia la ayuda a los demás tiene un efecto mayor en la intención de realizar donativos monetarios (Confiabilidad).

H2: La actitud hacia las organizaciones no lucrativas tiene un efecto mayor en la intención de realizar donativos monetarios (Seguridad).

La metodología aplicada en la investigación realizada captura información que concede un mayor nivel de respaldo a la propuesta presentada.

Para su desarrollo se utilizó un estudio de campo que permita obtener datos que serán 
usados posteriormente en conjunto como información para establecer la actitud e intención de los donantes.

\section{Técnicas de investigación}

Técnica de método multivariante por medio de:

- Análisis Factorial Confirmatorio para probar la validez y evaluar la confiabilidad de las escalas.

- Modelo de Ecuaciones Estructurales para probar las relaciones entre factores.

Las herramientas usadas fueron:

Encuesta: Se realizó un cuestionario dirigido hacia personas mayores de edad residentes de Guayaquil.

El cuestionario aplicado está dividido en seis secciones:

- La sección uno y dos se basan en las actitudes para ayudar a los demás (AHO) y las actitudes hacia las organizaciones benéficas (ACO) como determinantes distintos, pero a su vez afines en el comportamiento de los donadores. Estas dos secciones son tomadas del cuestionario de Actitudes que influyen en las donaciones monetarias a organizaciones benéficas de Webb, Green y Brashear (2000). La sección AHO está compuesta de cuatro elementos y cinco elementos para ACO. Las siguientes 4 secciones fueron planteadas con el objetivo de captar información relacionada a la intención, la preferencia respecto al destino de los donativos, la motivación, entre otras variables. Las siguientes secciones comprenden de preguntas cerradas.

Para el análisis de la investigación se ha considerado aplicar una escala de medición de Actitudes que influyen en las donaciones monetarias a organizaciones benéficas, implementada en el cuestionario realizo por Webb, Green, y Brashear (2000) y está compuesto en dos secciones. La primera sección es para medir la actitud para ayudar a los demás y la segunda sección es para medir la actitud hacia las organizaciones sin fines de lucro. Además, para medir la intención de donación se planteó una serie de preguntas basadas en el informe de El perfil del donante del año 2016 y se la dividió en cuatro secciones.

Para medir las 6 secciones del cuestionario se utilizó la escala de Likert de siete niveles que se sugirió en el modelo de Webb, Green, y Brashear, la cual oscila entre 1 (Totalmente en desacuerdo) a 7 (totalmente de acuerdo).

Para conocer la actitud que tiene las personas ante la ayuda social y las fundaciones se consideró el uso del modelo de medición; Actitudes que influyen en las donaciones monetarias a organizaciones benéficas realizada por Webb, Green, y Brashear (2000).

En la presente investigación la población se tomó, considerando el total de habitantes de la ciudad de Guayaquil hasta el 2019, según el Institutito Nacional de Estadística y Censo (INEC), siendo una población grande de medir se procedió a sacar una muestra significativa y se aplicó un muestreo no probabilístico por conveniencia. Tamaño de la población: $\mathrm{N}=$ 2’698077 y tamaño de la muestra: $n=390$

El cuestionario fue elaborado en la plataforma digital Encuesta Fácil, está plataforma es de fácil manejo y acceso para las personas encuestadas. El cuestionario se difundió en las principales redes sociales como WhatsApp, Facebook, Instagram y Twitter a través, de un enlace vía web. Permitiendo de esta manera que el usuario pueda acceder y contestar las diferentes secciones que ayudan en la recolección de información para la investigación.

La elaboración del análisis se usará herramientas y software estadísticos para el análisis de datos, dichos datos serán tomados en el mes de enero del presente año. Para el análisis de los datos obtenidos se aplicó el Modelo de Ecuaciones Estructurales por PLS, por medio del programa Smart PLS V3.

\section{RESULTADOS}

Según los datos recogidos del total de encuestados indica que el 54,6\% de encuestados pertenece al género femenino y el $45,4 \%$ de 
encuestados son del género masculino. Del total de encuestados se observa que la mayoría se encuentra en un rango de edad de 21 a 25 años con un porcentaje de $59,5 \%$, seguido del porcentaje $19,2 \%$ para el rango de edad de 18 a 20 años. Los que indicaría que el $78,7 \%$ de los datos recogidos fueron a personas jóvenes menores de 25 años. Todos los encuestados tenían un nivel de estudio, es decir ninguna persona no poseía estudio, el $58 \%$ de los encuestados tenían un nivel de estudio superior completo y el $40 \%$ tenía un nivel de estudio secundario completo. Más del $50 \%$ de los encuestados señaló que su estado civil es solero o soltera con un porcentaje de $86,8 \%$, seguido del estado civil casado o casada con un $8,4 \%$.

\section{Análisis de Variables \\ Motivación}

Los datos que muestran la tabla 3.4 son los valores del Coeficiente de Path de las variables que tuvo mayor peso en la investigación sobre la motivación de los ciudadanos guayaquileños al momento de donar. Dando como resultado del análisis que les motiva donar a las organizaciones si el motivo es cuando existe una emergencia o un evento catastrófico con un valor positivo significativo de 0,403 , para la motivación de donar a fundaciones cuando estas se encargan de ayudar a los demás con un valor positivo significativo de 0,384 y la motivación cuando se conoce el destino que se va a dar a dicha donación monetaria da un valor positivo y significativo de 0,376 . Siendo estos los valores más alto en la dimensión ayuda a organizaciones.

Tabla 1.

Análisis de Motivación

\begin{tabular}{lclll} 
& & $\begin{array}{l}\text { Motivación } \\
\text { a dar } \\
\text { donativos } \\
\text { para } \\
\text { ayudar a } \\
\text { a donar } \\
\text { completo } \\
\text { los demás. }\end{array}$ & $\begin{array}{l}\text { Motivación } \\
\text { a donaciones } \\
\text { cuando } \\
\text { existe una } \\
\text { emergencia/ } \\
\text { evento } \\
\text { catastrófico }\end{array}$ & $\begin{array}{l}\text { Motivación } \\
\text { a dar } \\
\text { donaciones } \\
\text { cuando se } \\
\text { conoce } \\
\text { como se } \\
\text { distribuye }\end{array}$ \\
\hline $\begin{array}{l}\text { Ayuda a } \\
\text { organizaciones }\end{array}$ & 0,395 & 0,384 & 0,403 & 0,376 \\
$\begin{array}{l}\text { Ayudar a los } \\
\text { demás }\end{array}$ & 0,293 & 0,326 & 0,314 & 0,299 \\
\hline
\end{tabular}

\section{Donación según su destino}

En la siguiente tabla 3.5 se indica los valores del Coeficiente de Path del segundo grupo de variables que tuvo mayor peso en la investigación sobre la intención según el destino de la donación de los ciudadanos guayaquileños. Dando como resultado del análisis de la intención a donar a las organizaciones si el destino es a las instituciones que ayuden a las personas que tengan una enfermedad grave o catastrófica con un valor positivo y significativo de $0,401 \mathrm{y}$ de igual valor la variable que indica la intención a donar a instituciones que se encargan de ayudar y proteger a los animales, con un valor positivo y significativo de 0,395 para la variable de intención a donar si la fundación se encarga de ayudar a los niños. Siendo estos los valores más alto en la dimensión ayuda a organizaciones.

Tabla 2.

Análisis de Intención según el destino

\begin{tabular}{lclll} 
& & $\begin{array}{l}\text { Intención a } \\
\text { realizar } \\
\text { donaciones a } \\
\text { instituciones } \\
\text { que ayuden } \\
\text { completo } \\
\text { a niños }\end{array}$ & $\begin{array}{l}\text { Intención a } \\
\text { realizar } \\
\text { donaciones a } \\
\text { instituciones } \\
\text { que ayuden a } \\
\text { las personas } \\
\text { con } \\
\text { enfermedades } \\
\text { catastróficas }\end{array}$ & $\begin{array}{l}\text { Intención a } \\
\text { realizar } \\
\text { donaciones } \\
\text { monetarias } \\
\text { para el } \\
\text { cuidado y } \\
\text { protección } \\
\text { de animales }\end{array}$ \\
\hline $\begin{array}{l}\text { Ayuda a } \\
\text { organizaciones }\end{array}$ & 0,395 & 0,396 & 0,401 & 0,378 \\
$\begin{array}{l}\text { Ayudar a los } \\
\text { demás }\end{array}$ & 0,293 & 0,359 & 0,34 & 0,401 \\
\hline
\end{tabular}

\section{Análisis de Fiabilidad y Validez de los constructos}

Los valores que se muestran en la tabla 3.4 representa a las dimensiones o componentes del modelo, su consistencia interna es representada por el Alfa de Cronbach, la Fiabilidad compuesta, la varianza extraída media (AVE) y el coeficiente de correlación de Spearman (Rho_A).

Tabla 3.

Fiabilidad y validez de constructo sobre la Intención de donar

$\begin{array}{cccc}\text { Alfa de } & \text { Rho_ } & \begin{array}{c}\text { Fiabilidad } \\ \text { Compuesta }\end{array} & \begin{array}{c}\text { Varianza } \\ \text { extraída } \\ \text { media } \\ \text { (AVE) }\end{array}\end{array}$




\begin{tabular}{lcccc}
\hline $\begin{array}{l}\text { Ayuda a } \\
\text { organizaciones }\end{array}$ & 0,834 & 0,835 & 0,889 & 0,668 \\
$\begin{array}{l}\text { Ayudar a los } \\
\text { demás }\end{array}$ & 0,862 & 0,867 & 0,906 & 0,707 \\
\end{tabular}

Para lograr la validez del modelo las dimensiones, el Alfa de Cronbach y la Fiabilidad compuesta debería tener al menos el valor mínimo requerido que es 0,7 que es el valor óptimo y como se puede observar en la tabla 3.2 y en la figura 3.5 se puede observar en el gráfico de barra el Alfa de Cronbach en cada variable los valores son mayor a dicho valor. Para la validez de la AVE el valor debe ser mayor o igual a 0,5 y se puede observar que dicho parámetro si se cumple en la tabla 3 en la columna de AVE, por la tanto los valores que dan validez a las dimensiones son positivos y significativos para el modelo.

\section{Validez Discriminante}

Según el criterio de Fornell y Larcker indica que la raíz cuadrada de la varianza media extraída de cada una de las variables debe ser mayor a la correlación que este mantenga con cualquier otro constructo. En la tabla 4 se puede observar los valores que se obtuvo al realizar el análisis. La diagonal restada representa a la raíz cuadrada de la varianza media extraída y los datos que están por debajo de esta diagonal representa la correlación de las variables latentes.

Tabla 4.

Validez Discriminante

\begin{tabular}{lccc}
\hline & $\begin{array}{c}\text { Ayuda a } \\
\text { organizaciones }\end{array}$ & $\begin{array}{c}\text { Ayudar a los } \\
\text { demás }\end{array}$ & $\begin{array}{c}\text { Intención a } \\
\text { ayudar }\end{array}$ \\
\hline $\begin{array}{l}\text { Ayuda a } \\
\text { organizaciones }\end{array}$ & $\mathbf{0 , 8 1 7}$ & \\
$\begin{array}{l}\text { Ayudar a los } \\
\text { demás }\end{array}$ & 0,364 & $\mathbf{0 , 8 4 1}$ & \\
\hline
\end{tabular}

\section{Análisis Coeficiente Path y Coeficiente de determinación $R^{\wedge} 2$}

En la siguiente figura se presenta el algoritmo PLS o estimación del modelo respecto a la intención a donar por parte de las personas entrevistadas, el resultado fue efectuado en el programa Smartpls basado en el modelo de
Ecuaciones Estructurales, el cual arrojo los valores factoriales de cada indicador, también se presentan los coeficientes de regresión o coeficientes Path.

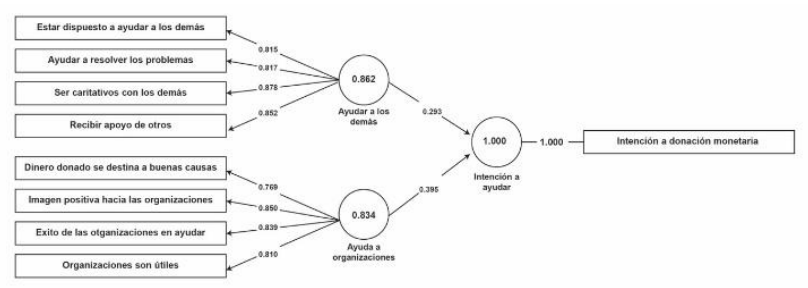

Figura2. Estimación del modelo Path sobre la Intención de donaciones

En el análisis de los resultados sobre la Intención a donar se presentaron los siguientes valores, en relación con los coeficientes Path. En el primer constructo, es decir Ayudar a los demás se obtuvo 0,293 y para Ayudar a las organizaciones fue de 0,395 , estos resultados son el total de los dos géneros, es decir los hombres y las mujeres tienen la intención de generar una ayuda monetaria a las organizaciones. Siendo la variable tres del constructo con el valor más alto $(0,85)$ es decir su nivel de aceptación en la Imagen favorable frente a las organizaciones benéficas, otra observación importante de resaltar es que la variable dos, fue eliminada porque no alcanzaba el valor mínimo de 0,7 .

Al unir las dos dimensiones arroja un valor según un coeficiente de Alfa de Cronbach que 1,0 y según el coeficiente de Path que nos indica el efecto de una variable sobre la otra variable y debe superar un valor mínimo de 0,20 para ser considerado valido y de esta manera evaluar el ajuste del modelo teórico que permite analizar la relación de dependencia de las variables.

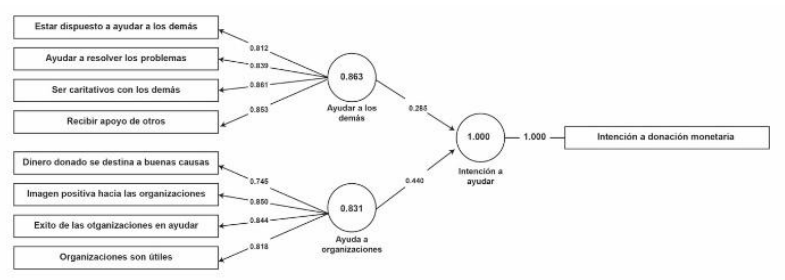

Figura 3. Estimación del modelo Path sobre la Intención de donaciones por parte de las mujeres guayaquileñas 
Los resultados sobre las Intención a donar por parte de las mujeres fueron los siguientes, en lo que respecta a los coeficientes Path para los Elementos tangibles fue de 0,440 en el segundo constructo, es decir tiene una aceptación mayor a ayudar a las organizaciones y sigue siendo la variable tres la más alta, es decir las mujeres ven de manera positiva el trabajo de las organizaciones benéficas, el primer constructo tiene un valor de 0,285 , siendo la variable tres de mayor valor, es decir tiene un puntaje de 0,861 en la pregunta que indica que las personas deberían ser más caritativas con lo demás.

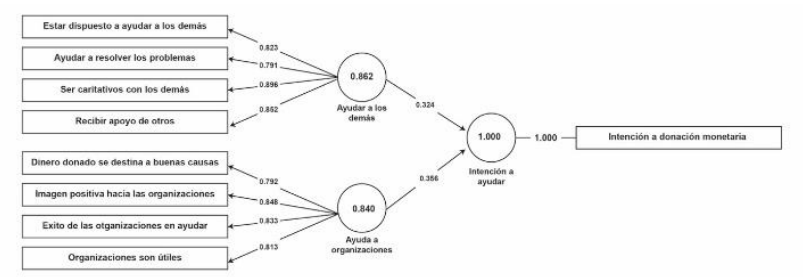

Figura 4. Estimación del modelo Path sobre la Intención de donaciones por parte de los hombres guayaquileñas

Estos resultados sobre las Intención a donar por parte de los hombres fueron los siguientes, en lo que respecta a los coeficientes Path para los Elementos tangibles fue de 0,356 en el segundo constructo, es decir tiene una aceptación mayor a ayudar a las organizaciones y sigue siendo la variable tres la más alta con un valor de 0,848 , es decir los hombres ven de manera positiva el trabajo de las organizaciones benéficas, el primer constructo tiene un valor de 0,356, siendo la variable tres de mayor valor, es decir tiene un puntaje de 0,861 en la pregunta que indica que las personas deberían ser más caritativas con lo demás.

\section{CONCLUSIONES}

En conclusión, se puede indicar mediante la técnica estadística PLS-SEM se pudo evaluar el proyecto de manera eficaz partiendo desde el modelo teórico realizado por cuatro hipótesis planteadas y dos constructos realizados sobre la actitud de realizar una ayuda social y la intención. De esta manera se validó el modelo, infiriendo que los guayaquileños tienen mayor intención a donar si es por medio de una organización benéfica.

Respecto al análisis de los datos, se tiene que los habitantes de la ciudad de Guayaquil tienen mayor aceptación a ayudar a través, de organizaciones benéficas a las diferentes causas. De igual manera, se obtuvo que la mayor motivación a dar donativos es cuando existe una emergencia o evento catastrófico o si su destino es a fundaciones que se encargan a ayudar a personas con enfermedades terminales o catastróficas. Por ultimo, el análisis reflejó que las mujeres tienen mayor aceptación en la intención de realizar donativos monetarios.

\section{Recomendaciones}

Finalmente, las organizaciones benéficas a pesar de tener una gran aceptación por parte del público no deben descuidar la imagen positiva que ya poseen. Por lo que se recomienda a las ONG trabajar en los diversos factores como, la comunicación de actividades que contribuyan a su misión.

Para mantener la confianza de las personas, las ONG deben ser transparentes con su financiamiento y el uso de los recursos monetarios captados. Por otra parte, las ONG deben enfocar sus esfuerzos comunicacionales y estrategias de marketing al grupo de personas cuyos intereses van acorde con la misión la organización, ya que esto les permitirá una mayor captación de recursos económicos.

\section{REFERENCIAS}

Aldamiz-Echeverría, G. D. (2003). Marketing en ONGs de desarrollo: para su aplicación práctica. Madrid: IEPALA.

Ajzen, I., \& Fishbein, M. (1998). Understanding attitudes and predicting social behavior. New York: Prentice-Hall.

Ander-Egg, E. (1986). Diccionario de Trabajo Social.

Wright, S. (1921). Un estudio sobre variabilidad genética, asociación de caracteres y análisis de 
coeficiente de ruta de caracteres morfológicos y de atribución de rendimiento del taro [Colocasia esculenta (L.) Schott].

Hair, J., Hult, G., Ringle, C., \& Sarstedt, M. (2017). A Primer on Partial Least Square Structural Equation Modeling (PLS-SEM). Estados Unidos, Califormina.

Melchor, A. (Noviembre de 2014). Researchgate. $\mathrm{O}$

Hair, J. F., Anderson, R. E., Tatham, R. L., \& Black, W. C. (1999). Análisis multivariante (Vol. 491). Madrid: Prentice Hall.

La Aduana. (s.f.). Servicio Nacional de Aduana del Ecuador. Obtenido de Servicio Nacional de Aduana del Ecuador: https://www.aduana.gob.ec/fundaciones-2/

Bonilla, E. (2008). Evidencias sobre el poder de la intención. Red de Revistas Cientifícas de América Latina, el Caribe, España y Portugal., 595-615.

La Hora. (13 de Marzo de 2005). Fundaciones sin control.

Bocanegra, L., Yesenia, M., \& Rodríguez, C. (7 de Julio de 2018). Repositorio Académico UPC. SUIOS. (2019). Sistema Unificado de Información de las Organizaciones Sociales.

García Elskamp, R. (1986). Intención e intencionalidad: estudio comparativo. Anales de filosofía, 147-150.

Fornell, C., \& Larcker, D. F. (1981). Evaluating structural equation models with unobservable variables and measurement error. Journal of marketing research, 18(1), 39-50.

Nunnally, J. C. (1994). Psychometric theory 3E. Tata McGraw-hill education.

Chin, W. W. (1998). The partial least squares approach to structural equation modeling. Modern methods for business research, 295(2), 295-336.
Burkardt, U. (2005). El concepto de fundraising y su aplicación a las donaciones monetarias procedentes de particulares. Barcelona: Centro de Investigación de Economía y Sociedad.

Mainar, I. G., \& Servós, C. M. (2002). Modelo familiar de donaciones monetarias a las organizaciones no lucrativas. ICE, Revista de Economía, (797).

Madero Cabib, I., \& Castillo, J. C. (2012). Sobre el estudio empírico de la solidaridad: aproximaciones conceptuales y metodológicas. Polis. Revista Latinoamericana, (31).

Wilkis, A., \& Instituto de Altos Estudios Sociales. (2008). Don, dinero y moral en el análisis de un circuito filantrópico. Instituto de Altos Estudios Sociales. Documentos de investigación del IDAES-UNSAM, 1, 1-10.

Kaztman, R. (2000). Notas sobre la medición de la vulnerabilidad social.

Sánchez-González, D., \& Egea-Jiménez, C. (2011). Enfoque de vulnerabilidad social para investigar las desventajas socioambientales: $\mathrm{Su}$ aplicación en el estudio de los adultos mayores. Papeles de población, 17(69), 151-185.

Fradua, I. A., Beloki, U., Sanz, A. D., \& Cabrera, M. S. (2017). Vulnerabilidad social percibida en contexto de crisis económica. RES. Revista Española de Sociología, 26(3), 17-39.

Molina, E. C., \& Cordova, J. D. (2014). El crédito de desarrollo humano asociativo en la economía social y solidaria de la provincia de TungurahuaEcuador. Analítika: revista de análisis estadístico, (7), 33-47.

TRELLES, I., \& Rodríguez, F. (2018). Diagnóstico de comunicación social: una dimensión de la vulnerabilidad del sistema de gestión de riesgos del Ecuador. Revista Espacios, 39(03).

Scharager, J., \& Reyes, P. (2001). Muestreo no probabilístico. Pontificia Universidad Católica de Chile, Escuela de Psicología, 1-3. 
Gracia, E., Herrero, J., \& Musitu, G. (1995). El apoyo social.

Beristain, C. M., \& Dona, G. (1999). Reconstruir el tejido social: un enfoque crítico de la ayuda humanitaria (Vol. 146). Icaria Editorial.

Reyes, G. E. (2012). Ayuda humanitaria y desarrollo: inclusión social y sostenibilidad. Compendium, 15(29), 67-96.

Maglieri, G. H. (1998). Organizaciones sin fines de lucro. FACES, 4(5), 95-127.

Gómez, M. H. (1998). La especificidad organizativa del tercer sector: tipos y dinámicas [Sociología de las organizaciones, Organizaciones sin fines de lucro]. Papers: revista de sociología, 163-196.

Picas Contreras, J. (2001). Papel de las Organizaciones No Gubernamentales y la crisis del desarrollo. Una crítica antropológica a las formas de cooperación, El. Universitat de Barcelona.

Vélez Zea, C. A. (2008). El impacto del recaudo por donación para causas sociales, en las actitudes y la intención de donar (Bachelor's thesis, Uniandes).

Hanisch Espíndola, H. (2010). La intención de donar. Revista de Estudios Histórico-Jurídicos, (2).

López-Sáez, M., \& Bustillos, A. (2008). Eficacia del uso de tácticas de influencia social en la propaganda de marketing social. Revista de psicología social, 23(1), 17-28.

Bowen Parada, Á. (2016). Estudio del comportamiento del consumidor con respecto a las" donaciones de los vueltos"

Rubilar, B. (2009). Donaciones para organizaciones con fines sociales y público. 